Journal of Theoretical and Applied Mechanics, Sofia, Vol. 47 No. 1 (2017) pp. 18-35

\title{
SPLINE PARAMETERIZATION OF COMPLEX PLANAR DOMAINS FOR ISOGEOMETRIC ANALYSIS
}

\author{
SANGAMESh GONDEgaOn, HARI K. VORUGANTI* \\ Department of Mechanical Engineering, National Institute of Technology, \\ Warangal, 506004 India
}

[Received 29 November 2016. Accepted 20 March 2017]

\begin{abstract}
Isogeometric Analysis (IGA) involves unification of modelling and analysis by adopting the same basis functions (splines), for both. Hence, spline based parametric model is the starting step for IGA. Representing a complex domain, using parametric geometric model is a challenging task. Parameterization problem can be defined as, finding an optimal set of control points of a B-spline model for exact domain modelling. Also, the quality of parameterization, too has significant effect on IGA. Finding the B-spline control points for any given domain, which gives accurate results is still an open issue. In this paper, a new planar B-spline parameterization technique, based on domain mapping method is proposed. First step of the methodology is to map an input (non-convex) domain onto a unit circle (convex) with the use of harmonic functions. The unique properties of harmonic functions: global minima and mean value property, ensures the mapping is bi-jective and with no selfintersections. Next step is to map the unit circle to unit square to make it apt for B-spline modelling. Square domain is re-parameterized by using conventional centripetal method. Once the domain is properly parameterized, the required control points are computed by solving the B-spline tensor product equation. The proposed methodology is validated by applying the developed B-spline model for a static structural analysis of a plate, using isogeometric analysis. Different domains are modelled to show effectiveness of the given technique. It is observed that the proposed method is versatile and computationally efficient.
\end{abstract}

KEY WORDS: Isogeometric analysis, analysis-suitable modelling, global parameterization.

\section{INTRODUCTION}

In conventional finite element analysis (FEA), the basis functions used for interpolation of field variable, are employed for interpolation of geometry as well, which results in poor approximation of geometric model. Computer aided design (CAD)

*Corresponding author e-mail: harikumar@gmail.com 
models use Non-Uniform Rational B-splines (NURBS) for the geometry representation. Great flexibility and precision for handling both analytic (surfaces defined by common mathematical formulae) and free-form shapes is the reason for the wide use of NURBS. So, when a CAD model is considered for simulation using FEA, the NURBS model is approximated by set of finite elements (line, triangle, tetrahedron, etc.). This process of dividing a CAD model into a set of elements is called meshing. $\mathrm{B}$-spline modelling is a continuous representation of the geometry, whereas mesh is the discrete representation, which results in loss of information. Apart from inaccurate geometric modelling, it is observed that, in analysis $80 \%$ of the total time is consumed by mesh generation [1]. To avoid the bottleneck of meshing, Hughes et al. [2] proposed a new method called Isogeometric Analysis (IGA), which integrates CAD and computer aided engineering (CAE). The idea of IGA is to use NURBS basis function for representation of both geometry and field variable. Geometry can be exactly represented in IGA, using NURBS basis functions. Due to integration of FEA and CAD, IGA simplifies the process of shape optimization [3].

One issue in IGA is to find the analysis suitable geometric B-spline model, which is still an open problem [4]. Any geometry can be represented exactly using NURBS with different parameterizations. But, the quality of parameterization possesses huge impact on the IGA results [5].

IGA requires parameterization of the whole domain, including the interior of the object. The model generated, using CAD packages contains information only about its boundaries. Modelling of parametric surface for a general non-convex region is still a challenging problem [4]. This is the inspiration to develop an efficient method for planar surface modelling, using domain mapping method [6]. In the proposed method, first we map the given non-convex domain to a circle (convex domain), using harmonic functions by domain mapping method to obtain entire domain parameterization. Then, the circle is re-parameterized to a rectangle for modelling of a B-spline surface.

The problem of surface modelling boils down to find the best set of control points for the given geometry, given the four boundary curves or a set of data points on the domain. B-spline modelling consists of two steps: surface parameterization and surface fitting. Both the steps are equally important for proper fitting of data points. B-spline modelling can be represented by [7]

$$
S(x, y)=\sum_{i=1}^{m} \sum_{j=1}^{n} P_{i j} R_{i j}(u, v),
$$

where $S(x, y)$ are data points to be fitted, $P_{i j}$ are the control points, $R_{i j}$ are the NURBS basis functions and $m \& n$ are the number of control points in the two 
parametric directions $u \& v$. Data points are the only known input for the surface construction.

There are three requirements that a CAD model should satisfy to be suitable for IGA: i) the mapping between physical and parametric domain should be bijective, which ensures no self- intersection, ii) the iso-parametric structure should be as orthogonal as possible and iii) iso-parametric lines of the B-spline surface should be uniformly distributed [8]. Since the proposed method is based on harmonic functions, all three conditions are inherently satisfied.

The remainder of the paper is organized as follows. Related work is discussed in Section 2, and the detailed methodology of mapping method is introduced in Section 3. Section 4 includes result and discussion, last section contains concluding remarks and scope of future work.

\section{STATE OF THE ART REVIEW}

The work related to IGA can be broadly divided into three categories: i) application of IGA in different areas; ii) use of different modelling techniques in IGA; iii) enhancing the efficiency and refinement techniques of IGA.

The work related to the applications of IGA includes solving problems in the field of static structural analysis, vibration analysis, thermal analysis, fluid-structure interaction, bio-mechanics, etc. Cottrell et al. [9] presented brief introduction of isogeometric analysis in the aspect of structural vibrations. They have solved different vibration models like rods, beams, plates and three dimensional solids. Cahn-Hilliard phase-field model is solved in [10] by Gomez et al. Adaptive time-stepping algorithm is used for effective solution of non-linear problems. In [11], fluid structure problems with interaction between incompressible fluid and non-linear elastic material are solved, using isogeometric analysis and the methodology is applied to analysis of arterial blood flow. Gondegaon et al. presented a detailed IGA implementation of static structural and modal analysis for one- and two-dimensional cases [12].

Work related to second category, i.e different modelling technique used for IGA are as follows. T-spline models are used for IGA, because of their ease and flexibility in geometric modelling in Escobar et al. [13], where adaptive tetrahedral mesh of parametric domain and B-spline is modelled, using mesh untangling and smoothing algorithm. A mapping-based tri-variate T-spline solid construction method was developed in Zhang et al. [14], for genus zero geometry, starting from a boundary surface triangulation. For finding the topology of the input geometry, saddle points are calculated by computing a harmonic scalar field. Input mesh is mapped to a polycube which is created using saddle points.

The proposed work comes under third category. In [15], the effect of smoothness of B-spline basis functions on accuracy of the IGA result is studied. Also, different 
refinement techniques with respect to IGA are explored on various numerical examples. Several parameterization methodologies using techniques like gradient based optimization, constraint optimization and variational harmonic method are proposed. Martin et al. [16] proposed a B-spline parameterization method for a triangular mesh, using harmonic function to construct a tri-variate B-spline volume. Triangle mesh of an unstructured data for representing boundary and also interior of the object for material attributes is taken as input. Harmonic functions are used for obtaining volumetric parameterization. The tri-variate B-spline is modelled, using an iterative fitting algorithm. Optimization based method for constructing a B-spline solid by considering positive Jacobian values is proposed by Wang et al. [17]. Fitting a Bspline volume, using optimization technique deals with a lot of variables and makes it computationally ineffective. To address this, they have divided the large optimization problem into number of small sub-problems. Kreisselmeier-Steinhauser function value is used for assigning a constraint to each sub-problem. Harmonic functions are used by Jüttler et al. [18] for parameterization of the computational domain. Parameterization of the domain is obtained by establishing bijective mapping, between original domain and the parameter domain (unit square or unit cube). A NURBS parameterization for swept volume is presented in Aigner et al. [19], using a variational approach, based on the boundary conditions.

In Gang Xu et al. [20], parameterization of the domain is obtained by solving a constraint optimization problem with injectivity sufficient conditions, as constraints. They have considered discrete Coon's patch for generating inner control points from a given four boundary B-spline curves. The optimized inner control points are calculated by minimizing the cost function, which includes stretch energy and bend energy. The effect of parameterization on three dimensional computational domains and injectivity conditions are proposed in Gang Xu et al. [21]. Discrete Coon's volume method is used for initial modelling of trivariate B-spline. They have proposed shape optimization technique for heat conduction problems with known exact solution. Similar work is extended for the isogeometric modelling of multi- block computational domain in [22]. In Pilgerstorfer et al. [23], the effect of domain parameterization and spacing of the knot vector on the numerical stability of the system is given. They have proposed a bound for condition number of the stiffness matrix, which depends on the mapping from physical to parameter domain.

It can be observed from literature, that the most of the parameterization methods used are based on the optimization technique, which is a tedious process. Some other related work includes projecting of the given geometry to the base surface to get a valid parameterization. Finding the base surface for many complicated geometry is not feasible.

Developing a B-spline representation of a complex surface is a challenging prob- 
lem, because there are two sets of unknowns namely parameter values and control points. The problem is intractable if both sets of unknowns are to be found together, as in the case of many approaches proposed so far. In contrast, separating the two sets of unknowns is the idea proposed in this work. First task is to develop a valid global parameterization for a given domain. Second, find the control points for the parameterized domain. The domain mapping method developed by Voruganti et al. [6] is used for domain parameterization.

\section{Methodology}

In the proposed planar surface modelling technique, first the given domain is parameterized by mapping it to a unit circle, using harmonic functions. Since B-spline modelling requires a rectangular topology, parameterized domain is re-parameterized to a rectangle. So the method is divided into three stages, a) parameterization of domain, b) mapping and re-parameterization c) surface fitting, which are explained below.

\subsection{PARAMETERIZATION OF 2D DOMAIN}

Domain mapping is the method of mapping a non-convex domain to a topologically equivalent convex domain. For a genus-zero domain, equivalent convex domain is a circle. Bijective mapping between an irregular domain and a circle is established, using harmonic functions. In the method, domain in the form of point cloud or four boundary curves are taken as input. Dirichlet boundary conditions are imposed, such that all the points on the boundary of the domain are assigned with high potential value $(\phi=1)$ and the shape centre, low potential value $(\phi=0)$. Laplace equation is solved, using finite difference method for computation of potential values inside the domain. Potential value at any point $P\left(x_{i}, y_{j}\right)$ in $k^{\text {th }}$ iteration is $\phi_{k}(x, y)$, then the next iteration is given by:

(2) $\phi_{k+1}\left(x_{i}, y_{j}\right)=\frac{\phi_{k}\left(x_{i+1}, y_{j}\right)+\phi_{k}\left(x_{i-1}, y_{j}\right)+\phi_{k}\left(x_{i}, y_{j+1}\right)+\phi_{k}\left(x_{i}, y_{j-1}\right)}{4}$.

This process gives an artificial potential field over the domain i.e, each point $P(x, y)$ has a potential value $\phi$. For a particular 2D domain, shown in Fig. 1a, corresponding iso-potential contours are developed, using the domain mapping method, as shown in Fig. 1b. Next step is the streamline tracking. Streamlines are gradient lines of potential. Streamlines start at the boundary and travels towards the shape centre by intersecting the iso-potential contours orthogonally. These lines intersect each contour at a unique angle, called streamline angle $(\theta)$. The streamlines are made up of a number of co-ordinate vectors, $X(t)$ originating from each boundary point at $t=0$ and travelling towards the shape centre as $t$ increases. If $\eta$ is a normalizing 
constant, streamlines tracking boils down to solving an ordinary differential equation

$$
X(t)=-\eta \nabla \phi[X(t)] .
$$

Streamline tracking provides another parameter, streamline angle $\theta$ at each point $P(x, y$,$) as shown in Fig. 1c. Now, each data point P(x, y)$ can be defined with two parameters, potential value $(\phi)$ and streamline angle $(\theta)$. This results in a complete mapping between the input domain $(x, y)$ and target domain (circle $(\phi, \theta))$, as shown in Fig. 1d. So, any point in an irregular domain can be mapped to a circle and viceversa, using these parameters.

The domain mapping method always results in a valid parameterization if not optimal, due to its bijective property [6]. Apart from global parameterization of the entire domain, this method is suitable for any non-convex domain.

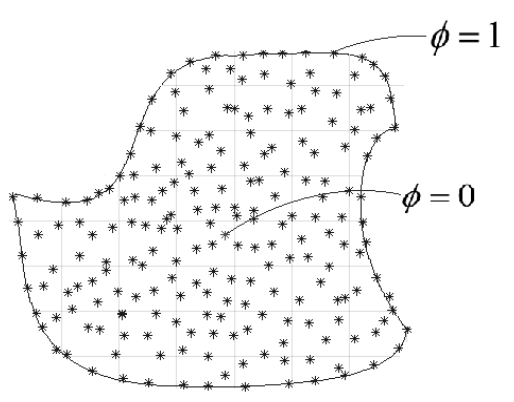

(a) Input domain

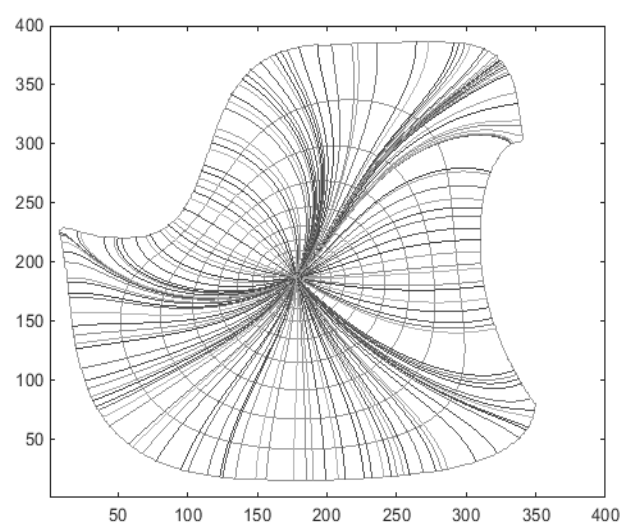

(c) Streamlines and contours

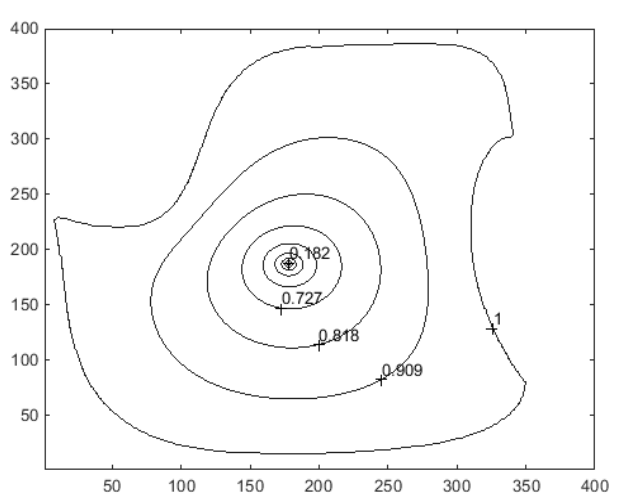

(b) Iso-potential contours

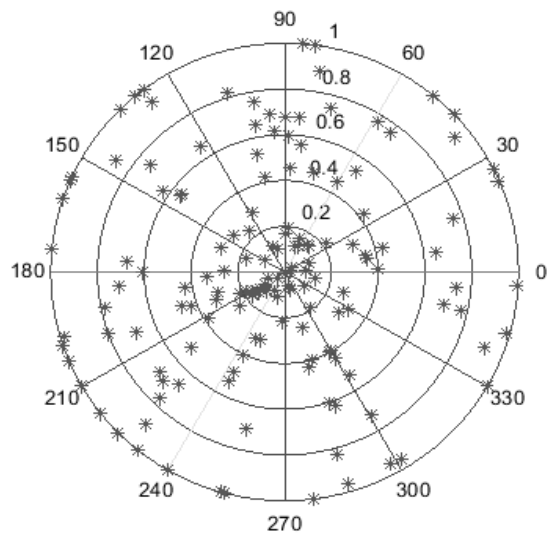

(d) Mapped data points in target domain

Fig. 1. Domain mapping steps. 


\subsection{MAPPING AND RE-PARAMETERIZATION}

Parameterized points from the previous step, as shown in Fig. 2a are in circle which cannot be used directly for surface fitting. For modelling of B-spline surface, the parameter domain should be rectangle, so these points should be mapped from circle to a rectangle.

In this step, streamline with $(\theta=0)$ is divided into $n / 2$ number of equidistant points, as shown in Fig. 2b, where $n$ is the number of data points in $u$ and $v$ direction. Potential value at each equidistant point is calculated, using interpolation. Contour at first point, $C_{1}$ on streamline, i.e $(\phi=1)$, is divided into four parts $\left(C_{11}, C_{12}, C_{13}\right.$, $C_{14}$ ) with each part of the contour, possessing $n$ number of data points, as shown in Fig. 2b. These four parts of the contour are equivalent to the four sides of the rectangle. Each of the four sides are iso-parametric lines representing $u=0, u=1$, $v=0$ and $v=1$, as shown in Fig. 2c. Similarly, second contour $C_{2}$ is considered at second point on streamline $(\theta=0)$ and is divided into four parts.

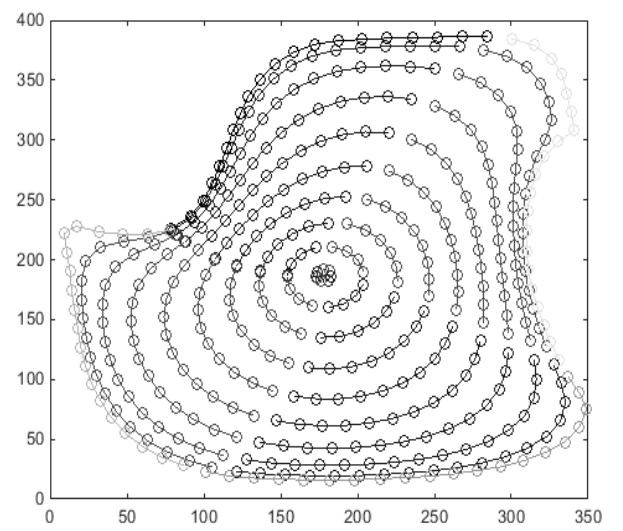

(a) $x-y$ domain

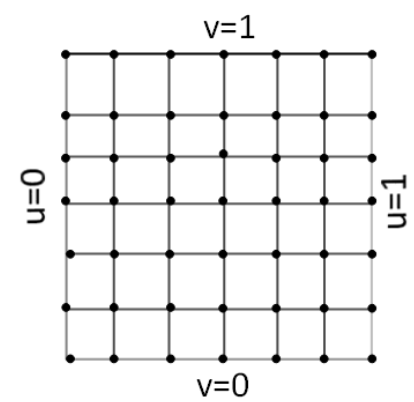

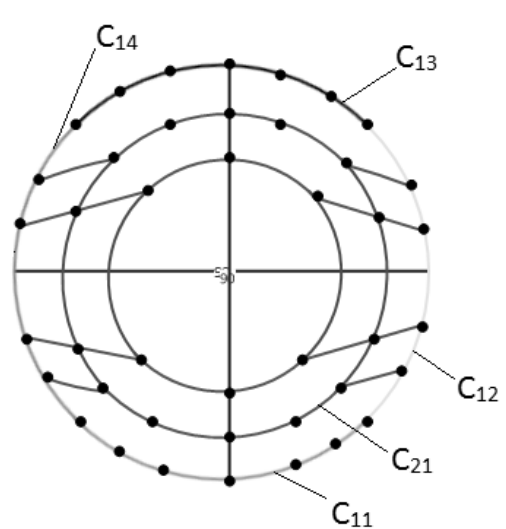

(b) $\phi-\theta$ domain

(c) $u-v$ domain

Fig. 2. Mapping from circle to rectangle. 
Each part of the second contour is divided into $(n-2)$ number of data points. The reason for reduction of point is that, end points of $C_{21}$ are second points of $C_{22}$ $(v=0)$ and $C_{24}(v=1)$. For $i^{t h}$ contour, each part (i.e $C_{i, 1}, C_{i, 2}, C_{i, 3}$ and $\left.C_{i, 4}\right)$ contains $(n-2(i-1))$ number of data points to get the required pattern, as shown in Fig. 2. Each data point is defined in a unique manner, using parameters $(\phi, \theta)$. In further calculation, for a given $(\phi, \theta)$, corresponding $(x, y)$ can be calculated, using the parameterization results.

Re-parameterization: $(\phi, \theta)$ to $(u ; v)$

Now, the order of the data points are known with the above process, a point in $(\phi, \theta)$ domain is assigned an iso-parametric line in $(u, v)$ domain i.e, one of the two parameter is fixed. For example, $C_{11}$ is mapped to $v=0$ line and have all points on $C_{11}$ are assigned with $v=0$. But, another parameter value is yet to be found. To achieve this, conventional parameterization methods like centripetal and chord-length can be used [24], because the data points are ordered.

\section{Determination of second parameter value}

Suppose the data points are $D_{0}, D_{1}, \ldots, D_{n}$, the distance between two adjacent data points is measured by $\left\|D_{k}-D_{k-1}\right\|^{a}$, rather than the conventional $\left\|D_{k}-D_{k-1}\right\|$, where $a=1 / 2$ for centripetal method. The length of the data polygon is

$$
L=\sum_{i=1}^{n}\left\|D_{i}-D_{i-1}\right\|^{a} .
$$

The ratio of the distance between $k^{t h}$ point and first point over the length of data polygon is given by

$$
L_{k}=\frac{\sum_{i=1}^{n}\left\|D_{i}-D_{i-1}\right\|^{a}}{L} .
$$

The parameters of the data points are given as:

$$
\begin{aligned}
& t_{0}=0 \\
& t_{k}=\frac{1}{L}\left(\sum_{i=1}^{n}\left\|D_{i}-D_{i-1}\right\|^{a}\right) . \\
& t_{n}=1
\end{aligned}
$$

If $a=1$, then centripetal method is reduced to chord-length method. All the data points are mapped from $(\phi \in(0,1)), \phi \in(0,2 \pi))$ to $(u, v) \in(0 ; 1)$, which enables B-spline surface fitting. 


\subsection{SURFACE FITTING}

Approximation method of surface fitting is chosen over interpolation method, because of its superiority in computational efficiency. Also, this method is more flexible and generates proper surface, even when the data points are incomplete or noisy. For a given set of data points, a B-spline surface model is obtained through direct matrix inversion method. The B-spline surface representation is given by

$$
S(u, v)=\sum_{i=1}^{n} \sum_{j=1}^{m} N_{i, p}(u) N_{j, q}(v) P_{i, j},
$$

where $N$ are basis functions of parameters $u$ and $v ; p$ and $q$ are the order of the Bspline surface; $n, m$ are the number of control points in $u, v$ parametric directions; $P_{i, j}$ are the control points of the B-spline surface. The number of data points that are given as input to find control point are $k$ and $l$. A data point, $s\left(u_{\alpha}, v_{\beta}\right)$ on the B-spline surface can be expressed as

(8)

$$
\begin{gathered}
s\left(u_{\alpha}, v_{\beta}\right)=N_{1}\left(u_{\alpha}\right) N_{1}\left(v_{\beta}\right) P_{11}+N_{1}\left(u_{\alpha}\right) N_{2}\left(v_{\beta}\right) P_{12}+\cdots++N_{1}\left(u_{\alpha}\right) N_{m}\left(v_{\beta}\right) P_{1 m} \\
+N_{2}\left(u_{\alpha}\right) N_{1}\left(v_{\beta}\right) P_{21}+N_{2}\left(u_{\alpha}\right) N_{2}\left(v_{\beta}\right) P_{22}+\cdots++N_{2}\left(u_{\alpha}\right) N_{m}\left(v_{\beta}\right) P_{2 m}+\cdots \\
+N_{n}\left(u_{\alpha}\right) N_{1}\left(v_{\beta}\right) P_{n 1}+N_{n}\left(u_{\alpha}\right) N_{2}\left(v_{\beta}\right) P_{n 2}+\cdots+N_{n}\left(u_{\alpha}\right) N_{m}\left(v_{\beta}\right) P_{n m} .
\end{gathered}
$$

When all the input data points $(k x l)$ are expressed in the form of Equation 8, it results in a linear systems of equations

(9)

$$
S=\left(\begin{array}{cccccc}
N_{1}\left(u_{1}\right) N_{1}\left(v_{1}\right) & \cdots & N_{1}\left(u_{1}\right) N_{m}\left(v_{1}\right) & N_{2}\left(u_{1}\right) N_{1}\left(v_{1}\right) & \cdots & N_{n}\left(u_{1}\right) N_{m}\left(v_{1}\right) \\
\cdots & \cdots & \cdots & \cdots & \cdots & \cdots \\
N_{1}\left(u_{1}\right) N_{1}\left(v_{l}\right) & \cdots & N_{1}\left(u_{1}\right) N_{1}\left(v_{l}\right) & N_{2}\left(u_{1}\right) N_{1}\left(v_{l}\right) & \cdots & N_{n}\left(u_{1}\right) N_{m}\left(v_{l}\right) \\
N_{1}\left(u_{2}\right) N_{1}\left(v_{1}\right) & \cdots & N_{1}\left(u_{2}\right) N_{m}\left(v_{1}\right) & N_{2}\left(u_{2}\right) N_{1}\left(v_{1}\right) & \cdots & N_{n}\left(u_{2}\right) N_{m}\left(v_{1}\right) \\
\cdots & \cdots & \cdots & \cdots & \cdots & \cdots \\
N_{1}\left(u_{k}\right) N_{1}\left(v_{l}\right) & \cdots & N_{1}\left(u_{k}\right) N_{m}\left(v_{l}\right) & N_{2}\left(u_{k}\right) N_{1}\left(v_{l}\right) & \cdots & N_{n}\left(u_{k}\right) N_{m}\left(v_{l}\right)
\end{array}\right)\left(\begin{array}{c}
P_{11} \\
P_{12} \\
\cdots \\
\cdots \\
\cdots \\
P_{n, m}
\end{array}\right) .
$$

The above Equation 9, can be written in a matrix form as

$$
[S]_{k \times l, 2}=[N]_{k \times l, n \times m}[P]_{n \times m, 2} .
$$

Equation 9 is solved to get the desired control points of the B-spline surface. The order of the required B-spline surface is chosen, based on the waviness in the data points. Open uniform knot vector is used for a given order of the B-spline surface fitting. Open knot vector ensures the B-spline surface will interpolate at the corner points. 
It is evident from the previous work, that most of the techniques used for surface construction method are based on optimization $[5,16,17]$. Optimization methods for surface construction deal with bigger number of variables like control points, knot vector and order of the B-spline surface and are also subjected to many nonlinear constraints. Solution of these non-linear optimization problems are computationally expensive and time consuming. Since the proposed method does not deal with optimization problem, it is more effective from all the previous work. The resulting B-spline surface, using proposed algorithm are shown in the next section.

\section{RESULTS AND DISCUSSION}

In the following section, various examples illustrating the performance of proposed method are discussed. Complete simulation work is carried out by developing code in Matlab 2015 a. Workstation used for the project is Dell Poweredge T320, Intel processor E5-2430 @2.20GHz and 16 GB RAM.

\subsection{IMPLEMENTATION ASPECTS}

Matlab implementation of the proposed method is explained briefly in the present section. The input, required for first stage i.e. domain mapping method is a set of point cloud. Each Cartesian co-ordinate of data points in the matrix form is taken as input. If the input is four b-spline boundary curves, point cloud is created, using inpolygon Matlab command.

Dirichlet boundary conditions are applied by assigning $(\phi=1)$ to the boundary points and $(\phi=0)$ at centre. Potential value at each point is calculated by solving Laplace equation by finite difference method using Matlab in-built solver. It is observed, that the variation of the potential value near the boundary of the domain is much smaller, where as it varies more towards the shape centre. Iso-potential contours are generated, using Matlab contour function. Streamlines, which are obtained by solving ordinary differential equation, ode 45 function is used, which is based on Runge-Kutta method with adaptive step-sizing. After establishing the complete bijective mapping, the points in the circle are mapped to the rectangle. Mapping between the two domains is carried out, using interpolation method and Matlab function interp is used.

\subsection{Test Problem}

To validate the proposed method, static structural problem of square plate with hole at centre under plane stress condition is considered. Equal traction force is applied to all the four boundaries of the square. Due to symmetry in material and loading conditions along both the axes, simulation is carried out only in one quadrant of the domain, as shown in Fig. 3. 


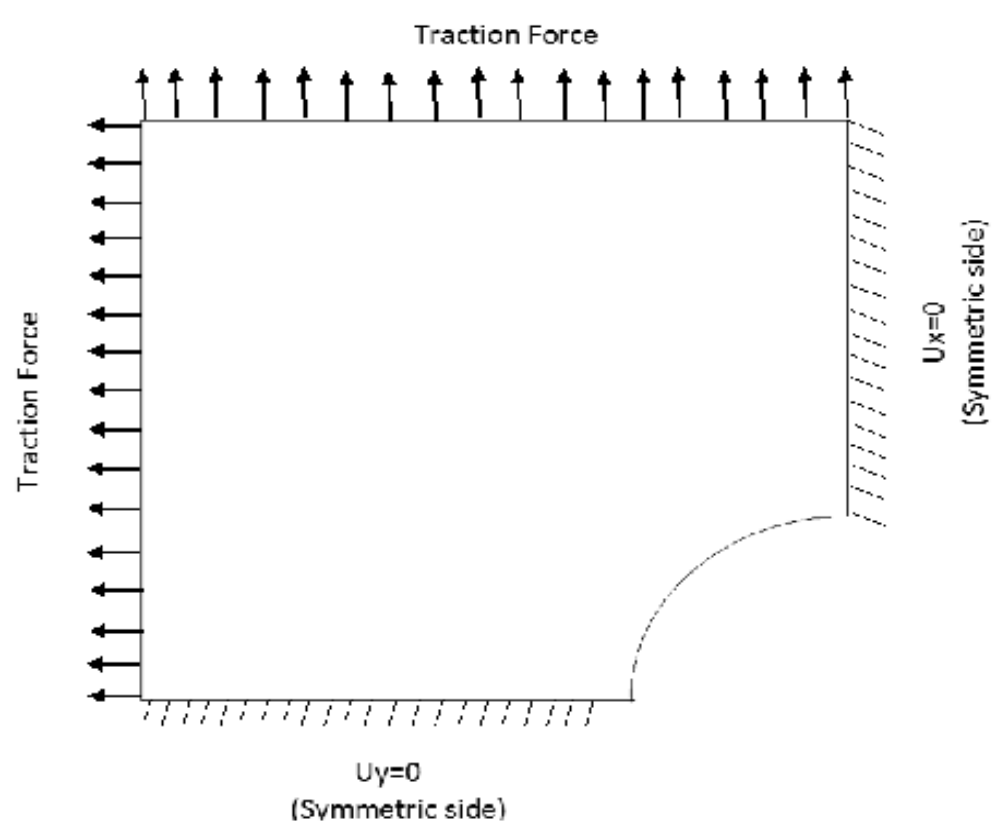

Fig. 3. Test problem.

The exact analytical solution for stress and displacement in x-direction over the domain in cylindrical co-ordinates is given in equations 11 and 12 [25]

(11) $U_{x}=-\frac{a}{8 \psi}\left[\frac{r}{a}(k+1) \cos (\theta)+\frac{2 a}{r}[(k+1) \cos (\theta)+\cos (3 \theta)]-\left(\frac{2 a}{r}\right)^{3} \cos (3 \theta)\right]$,

where $k=\frac{3-\mu}{1+\mu}$ and $\psi=\frac{E}{2(\mu+1)}$,

$$
\sigma_{x}=1-\left(\frac{a}{r}\right)^{2}[1.5 \cos (2 \theta)+\cos (4 \theta)]+1.5\left(\frac{a}{r}\right)^{4} \cos (4 \theta),
$$

where $(r, \theta)$ are the cylindrical co-ordinates, $E$ is the Young's modulus of the material, $\mu$ is the Poisson's ratio, $a$ is the hole radius and is the shear modulus.

The B-spline surface for the given problem is modelled, using the proposed method. Biquadratic surface with seven control points along each parametric direction is considered. The resulting iso-parametric net of B-spline surface is shown in Fig. 4. Simulation result for displacement in x-direction over the domain is shown in Fig. 5. The maximum displacement observed is $-4.8519 \times 10^{-5}$ and the maximum displacement calculated from the analytical solution is $-4.8969 \times 10^{-5}$. In Fig. 6, the absolute 


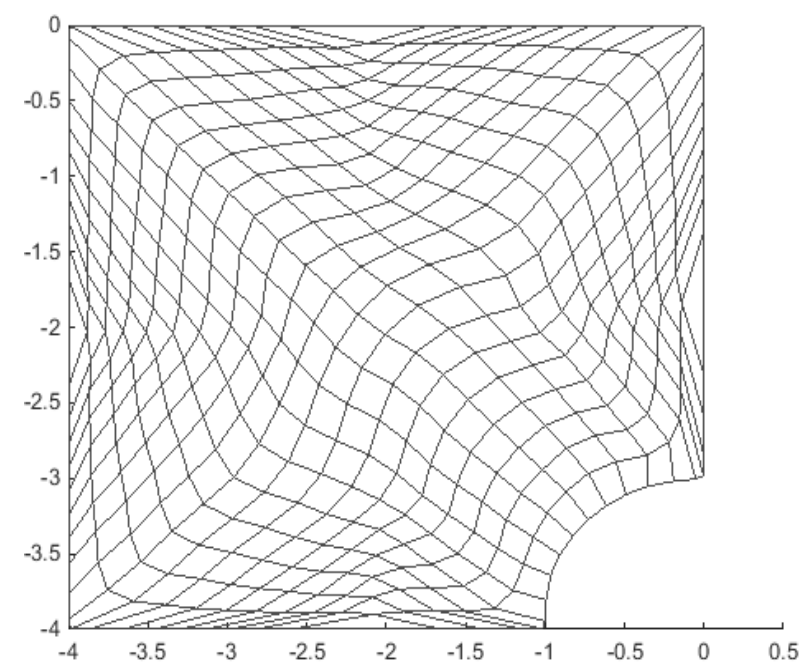

Fig. 4. B-spline model.

error between the simulation result and exact analytical solution is shown. Also, stress along $\mathrm{x}$-direction for the given problem and the corresponding error is shown in Figs. 7 and 8, respectively. From Figs. 6 and 8 it can be observed, that the simulation result is in good agreement with the exact solution.

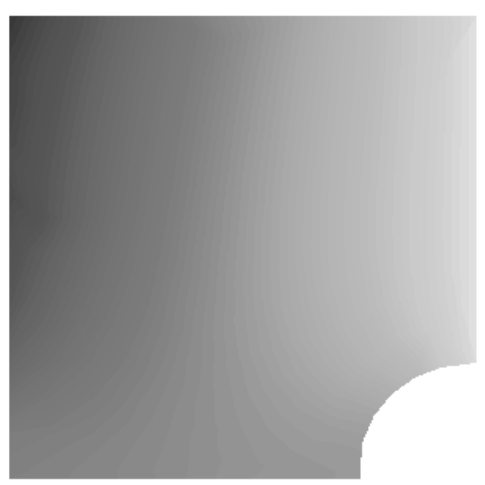

Fig. 5. Displacement in $x$-direction.

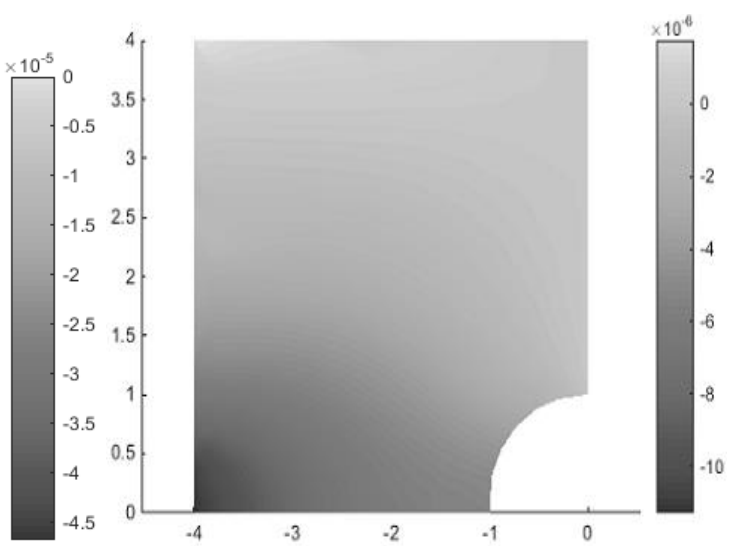

Fig. 6. Absolute error in displacement. 


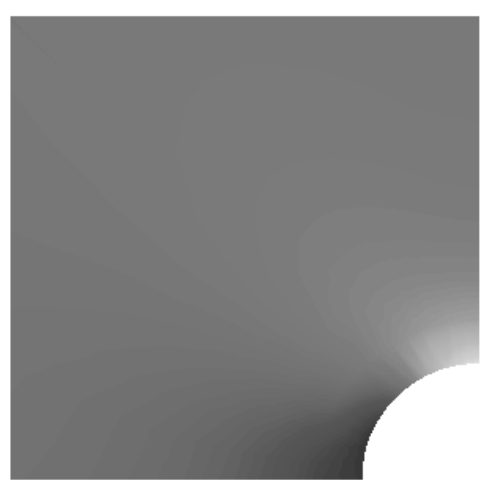

Fig. 7. Stress in $x$-direction.
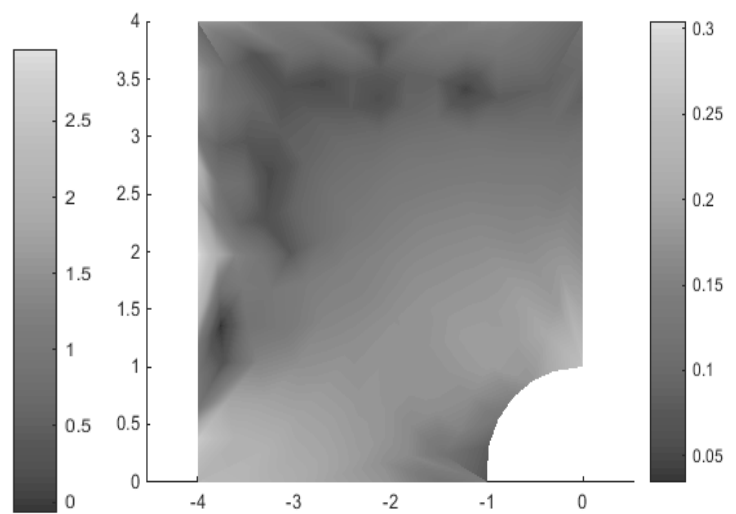

Fig. 8. Absolute error in stress.

\subsection{MORE NON-CONVEX CASES}

The proposed method is applied for two cases with non-convex geometry. For the first case, the boundary of the input domain, as shown in Fig. 9 is considered. The data points (900 in this case) of domain are shown in Fig. 10, which is the only input. This domain is mapped to circle and a valid parameterization is obtained. A subset of the 900 points is chosen, according to the re-parameterization algorithm, discussed in Section 3.2. This subset of data points is to determine the control points, using Equation 10. The input data points and the resulting control points (eight control points in each direction) are shown in Fig. 11. The iso-parametric net of the biquadratic B-spline surface model for the first case is shown in Fig. 12. Coon's surface

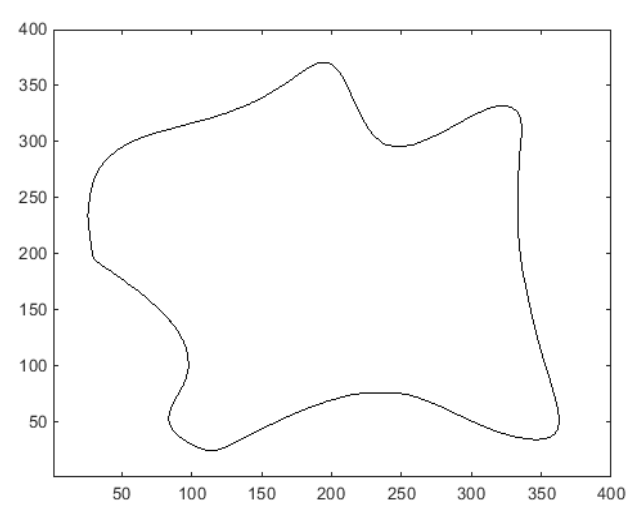

Fig. 9. Boundary of input domain.

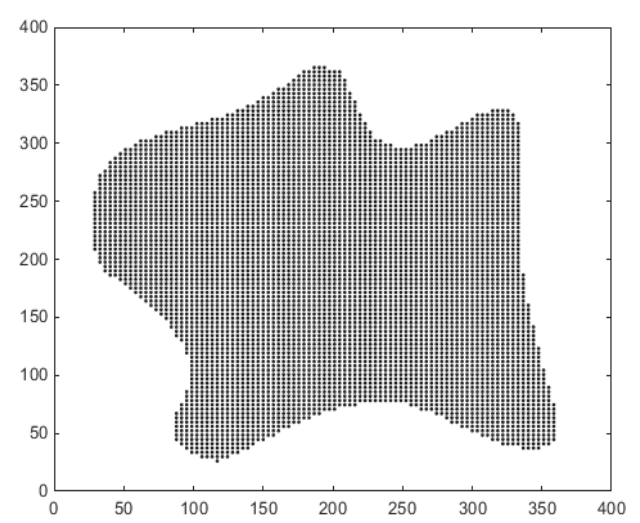

Fig. 10. Input domain with point cloud. 


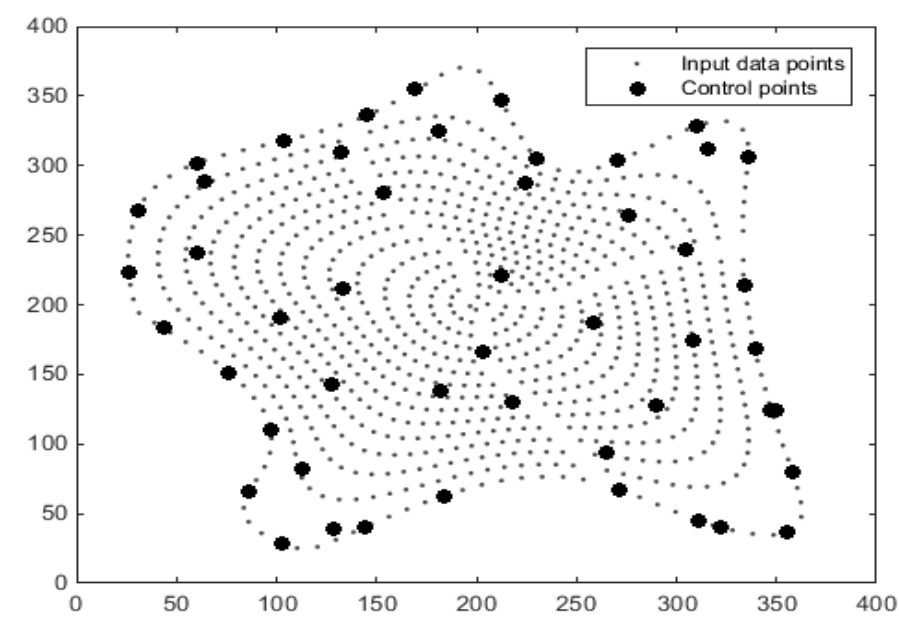

Fig. 11. Data points and control points.

for the same geometry is also generated, using discrete Coon's method for comparing the parameterization. Figure 14 shows the resulting Coon's parameterization. Coon's surface has self-intersection at top-right corner the magnified view of that part is shown in Fig. 15. The corresponding magnified view of iso-parametric net is shown in Fig. 13. It can be observed from Fig. 13, that the iso-parametric net modelled, using proposed method has no self-intersection, which is desirable for the isogeometric analysis.

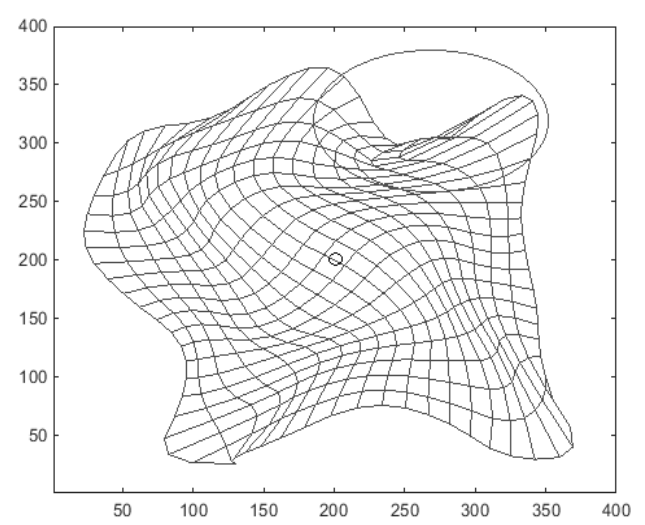

Fig. 12. Iso-parametric net for $1^{\text {st }}$ case.

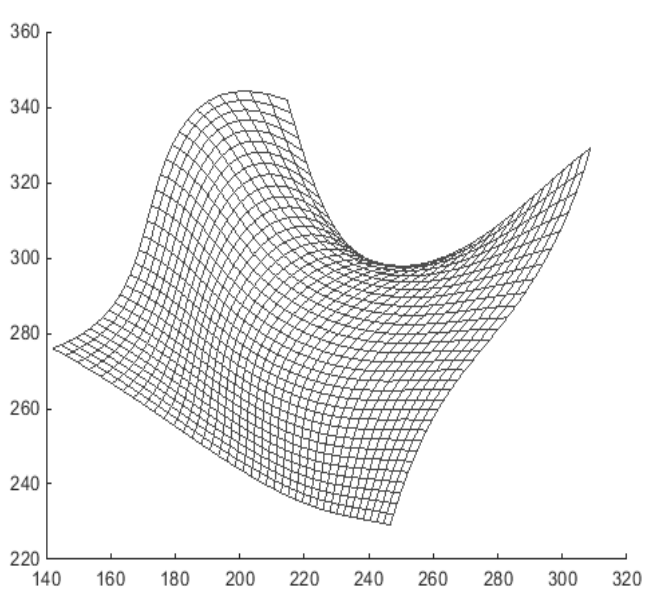

Fig. 13. Magnified view of Fig. 12. 


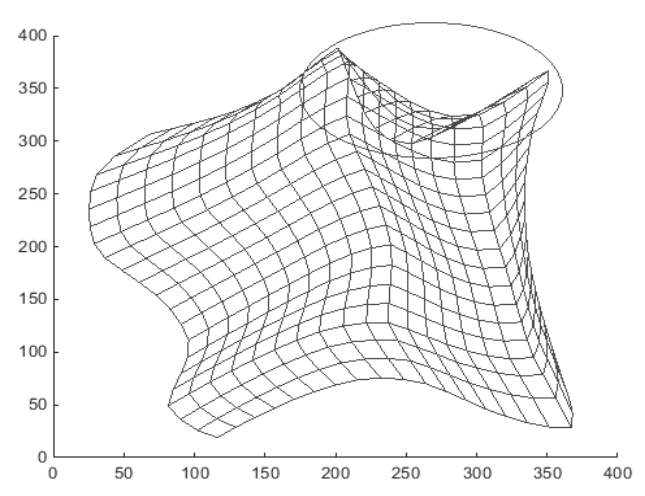

Fig. 14. Coon's surface for $1^{\text {st }}$ case.

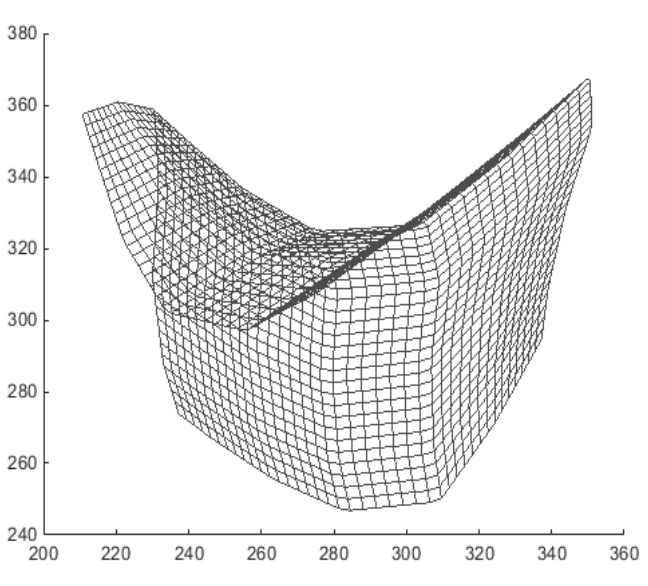

Fig. 15. Magnified view of Fig. 14.

Figures 16 and 17 show the iso-parametric net and Coon's parameterization, respectively for the second case. No self-intersection is observed in this case, as well. As pointed out in the literature [5], there is no quantitative analysis to assess the quality of the parameterization directly. It was also noted, that visually appealing parameterization is not necessarily good for analysis. The reported method always produces a valid parameterization and there will not be any self-intersections, because of the bi-jective properties of mapping method.

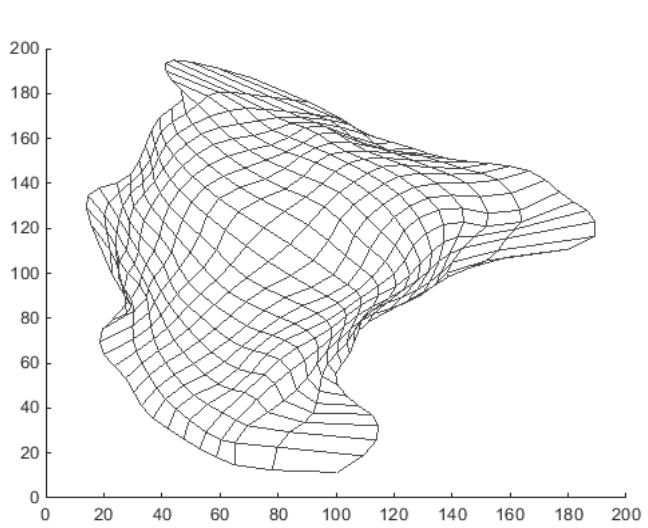

Fig. 16. Iso-parametric net for $2^{\text {nd }}$ case.

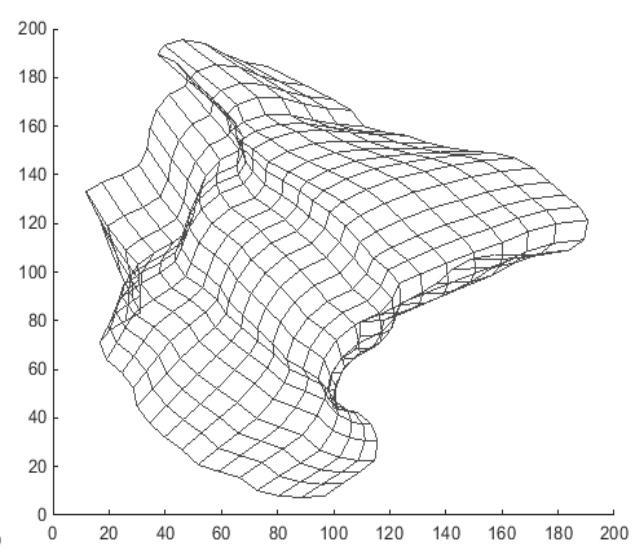

Fig. 17. Coon's surface for $2^{\text {nd }}$ case. 


\section{CONCLUSiOnS}

The quality of parameterization is a key issue in isogeometric analysis. A new method of parameterization, using domain mapping is proposed, which handles the geometrically complicated shapes with more effectiveness. A test problem is solved for the validation of the proposed method and different irregular shapes are modelled to show the effectiveness of the method. Unlike many previous ones, this method does not involve any optimization technique. Hence, the proposed method is computationally efficient. In extreme complicated shapes, if there is a poor approximation of geometry, one can refine the re-parameterization procedure presented in section 3.2, which is a part of the future work. Apart from this, the proposed method can be extended to volumetric parameterization and tri-variate B-spline modelling, as well.

Unlike in $\mathrm{CAD}$, the issue of domain parameterization arises in analysis, because of the need of information of interior of the domain, apart from boundary discretization. But, in some applications like shape optimization using IGA, only boundary discretization is required, because shape optimization is a boundary phenomenon. This method is called isogeometric analysis boundary element method (IGABEM), which possesses some advantage over conventional IGA for some particular sub-set of applications [25]. The case of 2-D shape optimization, using IGA is simpler, as it requires only 1-D parameterization of B-spline curve. But for the case of 3-D shape optimization, the input will be a boundary surface of the domain and hence bi-variate B-spline representation of boundary surface is required. Proposed method can be applied in this direction, also.

\section{REFERENCES}

[1] Hughes, T. J., J. A. Cottrell, Y. Bazilevs. Isogeometric Analysis: Cad, Finite Elements, Nurbs, Exact Geometry and Mesh Refinement. Computer Methods in Applied Mechanics and Engineering, 194 (2005), No. 39, 4135-4195.

[2] Cottrell, J. A., T. J. Hughes, Y. BAZILEvs. Isogeometric Analysis: Toward Integration of CAD and FEA, John Wiley \& Sons, 2009.

[3] Wall, W. A., M. A. Frenzel, C. CYron. Isogeometric Structural Shape Optimization. Computer Methods in Applied Mechanics and Engineering, 197 (2008), No. 33, 2976-2988.

[4] Brovka, M., J. López, J. Escobar, J. Cascón, R. Montenegro. A New Method for T-spline Parameterization of Complex 2d Geometries. Engineering with Computers, 30 (2014), No. 4, 457-473.

[5] Cohen, E., T. Martin, R. Kirby, T. Lyche, R. Riesenfeld. Analysis-aware Modelling: Understanding Quality Considerations in Modelling for Isogeometric Analysis. Computer Methods in Applied Mechanics and Engineering, 199 (2010), No. 5, 334-356. 
[6] Voruganti, H. K., B. Dasgupta, G. Hommel. Harmonic Function based Domain Mapping Method for General Domains. WSEAS Transactions on Computers, 5 (2006), No. 10, 2495-2502.

[7] Piegl, L., W. Tiller. The NURBS Book, Springer Science \& Business Media, 2012.

[8] Xu, G., B. Mourrain, A. Galligo, T. Rabczuk. High-quality Construction of Analysis-suitable Trivariate Nurbs Solids by Reparameterization Methods. Computational Mechanics, 54 (2014), No. 5, 1303-1313.

[9] Cottrell, J., A. Reali, Y. Bazilevs, T. Hughes. Isogeometric Analysis of Structural Vibrations. Computer Methods in Applied Mechanics and Engineering, 195 (2006), No. 41, 5257-5296.

[10] Gomez, H., V. M. Calo, Y. Bazilevs, T. J. Hughes. Isogeometric Analysis of Cahn Hilliard Phase Field Model. Computer Methods in Applied Mechanics and Engineering, 197 (2008), No. 49, 4333-4352.

[11] Bazilevs, Y., V. CAlo, Y. Zhang, T. J. Hughes. Isogeometric Fluid-structure Interaction Analysis with Applications to Arterial Blood Flow. Computational Mechanics, 38 (2006), No. 4-5, 310-322.

[12] Sangamesh, G., H. K. Voruganti. Static Structural and Modal Analysis Using Isogeometric Analysis. Journal of Theoretical and Applied Mechanics, 46 (2016), No. 4, 36-75.

[13] escobar, J., J. Cascón, E. Rodríguez, R. Montenegro. A New Approach to Solid Modelling with Trivariate T-splines based on Mesh Optimization. Computer Methods in Applied Mechanics and Engineering, 200 (2011), No. 45, 3210-3222.

[14] Wang, W., Y. Zhang, L. LiU, T. J. Hughes. Trivariate Solid T-spline Construction from Boundary Triangulations with Arbitrary Genus Topology. Computer-Aided Design, 45 (2013), No. 2, 351-360.

[15] Cottrell, J., T. Hughes, A. Reali. Studies of Refinement and Continuity in Tsogeometric Structural Analysis. Computer Methods in Applied Mechanics and Engineering, 196 (2007), No. 41, 4160-4183.

[16] Martin, T., E. Cohen, M. Kirby. Volumetric Parameterization and Trivariate Bspline Fitting using Harmonic Functions, in: Proceedings of the 2008 ACM Symposium on Solid and Physical Modelling, ACM, 2008, 269-280.

[17] Wang, X., X. QIAN. An Optimization Approach for Constructing Trivariate B-spline Solids. Computer-Aided Design, 46 (2014), 179-191.

[18] Nguyen, T., B. JÜTtLer. Parameterization of Contractible Domains using Sequences of Harmonic Maps, in: Curves and Surfaces, Springer, 2012, 501-514.

[19] Aigner, M., C. Heinrich, B. Jüttler, E. Pilgerstorfer, B. Simeon, A.-V. VUONG. Swept Volume Parameterization for Isogeometric Analysis, Springer, 2009.

[20] Xu, G., B. Mourrain, R. Duvigneau, A. Galligo. Parameterization of Computational Domain in Isogeometric Analysis: Methods and Comparison. Computer Methods in Applied Mechanics and Engineering, 200 (2011), No. 23, 2021-2031. 
[21] Xu, G., B. Mourrain, R. Duvigneau, A. Galligo. Optimal Analysis-aware Parameterization of Computational Domain in 3d Isogeometric Analysis. Computer-Aided Design, 45 (2013), No. 4, 812-821.

[22] Xu, G., B. Mourrain, R. Duvigneau, A. Galligo. Analysis-suitable Volume Parameterization of Multi-block Computational Domain in Isogeometric Applications. Computer-Aided Design, 45 (2013), No. 2, 395-404.

[23] Pilgerstorfer, E., B. Jüttler. Bounding the Influence of Domain Parameterization and Knot Spacing on Numerical Stability in Isogeometric Analysis. Computer Methods in Applied Mechanics and Engineering, 268 (2014), 589-613.

[24] Saxena, A., B. Sahay. Computer Aided Engineering Design, Springer Science \& Business Media, 2007.

[25] Nguyen, V. P., S. Bordas, T. Rabczuk. Isogeometric Analysis: an Overview and Computer Implementation Aspects, arXiv Preprint arXiv: 1205.2129.

[26] Haojie, L., P. Kerfriden, S. Pierre, A. Bordas. Implementation of Regularized Isogeometric Boundary Element Methods for Gradient-based Shape Optimization in Two-dimensional Linear Elasticity. International Journal for Numerical Methods in Engineering, 106 (2015), 972-1017. 